\title{
Stressors and level of stress among different nursing positions and the associations with hyperlipidemia, hyperglycemia, and hypertension: a national questionnaire survey
}

\author{
Po-Ya Chang ${ }^{1}$, Shu-Ti Chiou ${ }^{2,3}$, Wen-Yen Lo ${ }^{4}$, Nicole Huang ${ }^{5}$ and Li-Yin Chien ${ }^{6 *}$ (D)
}

\begin{abstract}
Background: Nurses are faced with varying job stressors depending on their positions and duties. Few previous studies have compared job stress and related chronic conditions among different nursing positions. The objectives were to compare job stressors among clinical registered nurses, nurse practitioners, and head nurses and explore the impact of job stressors and stress level on hyperlipidemia, hyperglycemia, and hypertension.

Methods: Secondary data extracted from a survey of health-care workers conducted from May to July 2014 across 113 hospitals in Taiwan was used. This analysis included 17,152 clinical registered nurses, 1438 nurse practitioners, and 2406 head nurses. Socio-demographic characteristics, job stressors, stress levels, and hyperlipidemia, hyperglycemia, and hypertension variables were extracted.

Results: Perceived stressors differed among clinical registered nurses, nurse practitioners, and head nurses, but overall stress level did not. Nurse practitioners and head nurses showed significantly higher prevalence of hyperlipidemia, hyperglycemia, and hypertension than clinical registered nurses. Higher stress levels, age, body mass index, work hours, and caring for family members were positively associated with hyperlipidemia, hyperglycemia, and hypertension. After adjustment for these variables, risk of hyperlipidemia, hyperglycemia, and hypertension did not differ across the nursing positions.

Conclusions: Although stressors vary by different nursing positions, overall stress level does not. Hyperlipidemia, hyperglycemia, and hypertension are related to stress level, age, body mass index, weekly working hours, and caring for family members. Hence, alleviating job stress and avoiding long working hours are likely to reduce the risk of hyperlipidemia, hyperglycemia, and hypertension in nurses.
\end{abstract}

Keywords: Hypertension, Hyperglycemia, Hyperlipidemia, Nurses, Nursing, supervisory, Head nurses, Nurse practitioners, Job stress

\footnotetext{
* Correspondence: lychien@nycu.edu.tw

${ }^{6}$ Institute of Community Health Care, College of Nursing, National Yang Ming

Chiao Tung University, Yang-Ming Campus, 155 Li-Nong Street, Section 2,

Bei-Tou, Taipei 11221, Taiwan

Full list of author information is available at the end of the article
} 


\section{Introduction}

In many countries, nursing is considered as a highly stressful occupation [1], and high work stress is a longterm problem in the health-care industry $[1,2]$. Studies have found work stress to be related to hyperlipidemia, hyperglycemia, and hypertension [3-5]; however, the strength of these associations across different nursing positions has not been well-established. In the healthcare system, nursing staff perform various roles and functions, with their specific responsibilities depending on their positions [6]; thus, the stressors and work stress experienced by nurses may differ depending on their job responsibilities.

Encountering stress from various sources at work may cause distress symptoms, and inadequate treatment of these symptoms could result in the development of physiological and/or mental diseases [7]. Over the past 20 years, many studies have examined the correlation between work stress and hyperlipidemia, hyperglycemia, and hypertension $[3,5]$, as well as the possibility of such stress increasing the risk of coronary heart disease [8].

Several cross-sectional studies have reported that work stress is a risk factor for hyperlipidemia [3, 9, 10]. Several studies, including laboratory data papers [11], casecontrol studies [12], and meta-analyses [4, 13] have shown that stress increases blood glucose levels. For example, Kivimäki and Kawachi (2015) examined 27 cohort studies on work stress and type 2 diabetes and found that high work stress is a risk factor for type 2 diabetes [4]. Systematic reviews and meta-analyses have shown that work stress is related to hypertension $[5,14$, 15]. Notably, Landsbergis (2013) examined 22 crosssectional studies and found work stress to be significantly related to hypertension [14]. However, despite the above findings, the association between job stress and conditions of hyperlipidemia, hyperglycemia, and hypertension has not been examined and compared across different nursing positions.

Nurses account for a large proportion of the personnel in the health-care industry. They play a crucial role in clinical care, and have a wide variety of responsibilities [16]. However, nurses were found to experience moderate to high work stress [17, 18], with their work stressors including workloads, shifts, role conflicts, interpersonal relationships, workplace violence, insufficient preparation, and uncertainty regarding treatment results [1, 2, 19].

The United States (US)' health-care system features four types of advanced-practice registered nurses: certified nurse practitioners (NPs), certified registered nurse anesthetists, clinical nurse specialists, and certified nurse midwives [20]. NPs' primary responsibilities include patient care, health education, medical coordination, and medical quality control [20]. In the Taiwanese medical system, clinical nursing staff can be divided into clinical registered nurses (CNs), NPs, and head nurses/nurse managers (HNs). In Taiwan, approximately $83 \%$ of nurses work in hospitals [21], with NPs performing much of the clinical work in hospitals; in 2018, there were 7685 practicing NPs in Taiwan [22]. NPs, who are supervised by physicians, were found to experience moderate work stress, originating from factors such as the $\mathrm{CN}$ to NP transition, low confidence, the need to meet the expectations of team members, and anxiety regarding the opinions of colleagues [23-25]. Meanwhile, HNs are responsible for the general operation of the entire ward and quality maintenance of patient care in the unit; HNs also commonly experience considerable stress during work [26], with one in six HNs experiencing work stress and job burnout [27]. Stressors for HNs include role conflicts, time pressure, workload, insufficient workplace support, insufficient leadership, rapid transformations of medical systems, and organizational limitations $[16,26]$.

Previous studies on work stress among nurses have compared job stress across hospital levels, work units, and qualifications [16, 28]; however, few studies have examined the level of perceived job-related stress, and the association of such stress with diseases, across different job positions. Therefore, this study aimed to compare job stressors among CNs, NPs, and HNs, and examine the impact of different job stressors and overall level of stress on the prevalence of hyperlipidemia, hyperglycemia, and hypertension among these nurses.

\section{Methods}

\section{Design and participants}

This secondary data analysis was performed using data sourced from a national cross-sectional study of fulltime health-care workers in Taiwan that was conducted from July to September 2014. This study invited those health facilities that ever jointed the Taiwan Health Promoting Hospital Network, ever participated in the previous similar survey in 2011, or ever applied for certification of health promoting hospital and healthcare institution to participate. Of the 157 health facilities invited, 113 agreed to participate, including 13 medical centers, 59 district hospitals, 32 regional hospitals, eight public-health centers, and one nursing home. After obtaining approval from the hospitals, envelopes with consent forms and questionnaires were distributed to each of the hospital. Health-care workers were asked to return the signed consent forms and filled questionnaires using the provided two separated and sealed envelopes to the data collection site at each hospital. Overall, 111,110 questionnaires were distributed, 89,014 filled questionnaires were returned, and 75,841 valid questionnaires were collected (valid response rate $=68.3 \%$ ). The 
dataset with valid records were released to the research team by the Taiwan Health Promotion Administration. For the present study, questionnaires answered by nonnursing staff (physicians, pharmacists, and other medical professionals) and non-clinical staff (administrative staff, medical laboratory personnel, laborer, and others) were excluded, remaining 20,996 anonymous questionnaires completed by full-time clinical nurses, which formed the analytical sample for this secondary data analysis. Among these nurses, 2406 (11.5\%) were HNs, 1438 (6.8\%) were NPs, and 17,152 (81.7\%) were CNs. Details of the study design are presented elsewhere [29].

\section{Measures}

Data were collected through a structured questionnaire that focused on the physical and mental health, and safety of the participants. Prior to the data collection, six experts reviewed and revised the questionnaire content over two meetings, and a pilot test was performed on 10 health-care workers to ensure its clarity and readability.

Hyperlipidemia, hyperglycemia, and hypertension were measured using yes/no questions regarding the presence of any of those chronic conditions. The study variables related to hyperlipidemia, hyperglycemia, and hypertension included socio-demographics (age, gender, and marital status), work experience (years of work experience, work unit, job position), medical history and chronic diseases, self-reported weight and height, and family-care needs. Family-care needs was measured by a question enquiring "current family-care needs" with four items -- (1) self or spouse being pregnant, (2) having children at preschool age, (3) having children in elementary school and/or high school, (4) having other family member who is in need of care. Any positive answer to the four items was coded as yes, otherwise it was coded as no.

Sources of work stress and level of perceived stress were measured by a scale of 23 items. A five-point Likert scale (1-5) was used to measure level of perceived stress; higher scores indicated greater stress, given a summary score ranged from 23 to 115 points. The reliability and validity of the scale have been described previously [29]. The scale explained $62.13 \%$ of the variance, and the internal consistency was 0.93 [18], supporting construct validity and reliability of the scale. Meanwhile, the 23 items represented eight potential sources of job stress: (1) evaluation and policy; (2) colleagues, higher-level officers, and extraoccupational duties; (3) supervisor of own department; (4) work content and opinions of individuals from other departments; (5) lack of work leadership, security, and equality; (6) impact of and fatigue from work performance; (7) promotion; and (8) others.

\section{Data analysis}

Statistical analysis was performed using SPSS 23.0 (IBM Corp., Armonk, NY, USA). Means and standard deviations were used to describe continuous variables, and frequencies and percentages were used to describe categorical variables and the overall distribution of the sample. Analysis of variance (ANOVA) was used to compare the level of stress among the three different nurse positions. If a significant difference was found, the Scheffé post-hoc test was conducted for pairwise comparisons. Further, logistic regression models were applied to examine the factors associated with the presence of any of the chronic conditions including hyperlipidemia, hyperglycemia, and hypertension.

\section{Results}

Table 1 shows the characteristics of the 20,996 nurses. HNs had the highest mean age (39.1 years, standard deviation $[S D]: 7.09)$, followed by NPs (37.6, $S D: 5.55)$, and CNs (31.7, SD: 0.66). Most participants (98\%) were women. Over one quarter (26.2\%) were overweight or obese (body mass index $[\mathrm{BMI}] \geq 24)$. Over half $(60 \%)$ of the NPs and HNs were married; most CNs (>60\%) were never married. HNs had the highest mean number of years of work experience, at 20.35 (SD: 9.75), followed by NPs at 15.14 (SD: 9.72) and CNs at 11.96 (SD: 10.44). The general ward was the most-represented work unit (42.7\%). HNs had the highest mean number of work hours per week $(50.72, S D: 12.84)$, followed by NPs (49.98, SD: 12.18) and CNs (47.49, SD: 11.65). The CN group had the highest proportion of members who had family-care needs $(49.6 \%)$.

The prevalence among the sample of any of the constituents of hyperlipidemia, hyperglycemia, and hypertension was $9.5 \%$ (Table 1). Of these, most had hypertension $(n=931,4.8 \%)$, followed by hyperlipidemia $(n=898,4.6 \%)$ and hyperglycemia $(n=541,2.8 \%)$. The prevalence of hyperlipidemia, hyperglycemia, and hypertension was highest among HNs (14.8\%), followed by NPs (12.2\%) and CNs (8.5\%).

An ANOVA (Table 2) showed no significant difference among the nursing positions regarding scores for overall perceived work stress. However, analysis of stressors showed significant differences among the three nursing positions. The mean levels of evaluation- and policyrelated stress were significantly higher among HNs than $\mathrm{CNs}$ and NPs. Meanwhile, scores for stress from colleagues, higher-level officers, and extra-occupational $\mathrm{du}$ ties were significantly higher for HNs than NPs and CNs. NPs showed significantly higher scores for stress from work content and the opinions of individuals from other departments when compared to HNs and CNs. NPs showed the highest mean score for stress from inadequate work leadership, security, and equality, followed by $\mathrm{CNs}$ 
Table 1 Characteristics of the participating nurses

\begin{tabular}{|c|c|c|c|c|c|}
\hline Variables & $\begin{array}{l}\text { All } \\
(n=20,996) \\
n(\%)\end{array}$ & $\begin{array}{l}\text { Clinical } \\
\text { nurses } \\
(n=17,152) \\
n(\%)\end{array}$ & $\begin{array}{l}\text { Nurse practitioners } \\
(n=1438) \\
n(\%)\end{array}$ & $\begin{array}{l}\text { Head } \\
\text { nurses } \\
(n=2406) \\
n(\%)\end{array}$ & $p$ value \\
\hline \multicolumn{6}{|l|}{ Age (years) } \\
\hline$<26$ & $4093(20.4)$ & $4062(24.8)$ & $15(1.1)$ & $16(0.7)$ & \multirow[t]{5}{*}{$<0.001^{*}$} \\
\hline $26-35$ & 9107 (45.5) & $7832(47.9)$ & $542(39.6)$ & $733(31.8)$ & \\
\hline $36-45$ & $5089(25.4)$ & $3328(20.4)$ & $704(51.5)$ & $1057(45.9)$ & \\
\hline $46-55$ & $1586(7.9)$ & $1017(6.2)$ & $102(7.5)$ & $467(20.3)$ & \\
\hline$>55$ & $143(0.7)$ & $108(0.7)$ & $4(0.3)$ & $31(1.3)$ & \\
\hline \multicolumn{6}{|l|}{ Gender } \\
\hline Male & $412(2.0)$ & $311(1.9)$ & $65(4.7)$ & $36(1.6)$ & \multirow[t]{2}{*}{$<0.001^{*}$} \\
\hline Female & $19,883(98.0)$ & $16,279(98.1)$ & $1320(95.3)$ & $2284(98.4)$ & \\
\hline \multicolumn{6}{|l|}{ BMI $\left(\mathrm{kg} / \mathrm{m}^{2}\right)$} \\
\hline$<18.5$ & $2124(11.9)$ & $1894(12.9)$ & $91(7.6)$ & $139(6.8)$ & \multirow[t]{3}{*}{$<0.001^{*}$} \\
\hline $18.5-23.9$ & $11,077(62.0)$ & $9058(61.9)$ & $792(65.7)$ & $1227(60.2)$ & \\
\hline$>24$ & $4678(26.2)$ & $3684(25.2)$ & $322(26.7)$ & $672(33.0)$ & \\
\hline \multicolumn{6}{|l|}{ Marital status } \\
\hline Never married & $10,796(54.8)$ & $9672(60.1)$ & $437(32.3)$ & $687(30.3)$ & \multirow[t]{3}{*}{$<0.001^{*}$} \\
\hline Married & 8390 (42.6) & $6048(37.6)$ & $846(62.6)$ & $1496(66.0)$ & \\
\hline Divorced or widowed & $516(2.6)$ & $365(2.3)$ & $68(5.0)$ & $83(3.7)$ & \\
\hline \multicolumn{6}{|l|}{ Work experience (years) } \\
\hline$<5$ & $6719(35.5)$ & $6286(40.9)$ & $303(23.1)$ & $130(5.7)$ & \multirow[t]{4}{*}{$<0.001^{*}$} \\
\hline $5-9$ & $328(1.7)$ & $240(1.6)$ & $24(1.8)$ & $64(2.8)$ & \\
\hline $10-14$ & 3045 (16.1) & $2206(14.4)$ & $284(21.7)$ & $555(24.5)$ & \\
\hline$\geq 15$ & $8852(46.7)$ & $6639(43.2)$ & $698(53.3)$ & $1515(66.9)$ & \\
\hline \multicolumn{6}{|l|}{ Work unit } \\
\hline OPD & $2584(12.3)$ & $2384(13.9)$ & $31(2.2)$ & $169(7.0)$ & \multirow[t]{5}{*}{$<0.001^{*}$} \\
\hline ER/ICU & $4736(22.6)$ & 3929 (22.9) & $269(18.7)$ & $538(22.4)$ & \\
\hline General ward & 8955 (42.7) & $7174(41.8)$ & $692(48.1)$ & $1089(45.3)$ & \\
\hline OR/DR & 2428 (11.6) & $2114(12.3)$ & $69(4.8)$ & $245(10.2)$ & \\
\hline Multiple units & $2293(10.9)$ & $1551(9.0)$ & $377(26.2)$ & $365(15.2)$ & \\
\hline \multicolumn{6}{|l|}{ Weekly work hours } \\
\hline$\leq 40$ & $5260(28.4)$ & $4538(30.1)$ & $238(18.9)$ & $484(22.5)$ & \multirow[t]{3}{*}{$<0.001^{*}$} \\
\hline $41-49$ & 8060 (43.6) & $6622(43.9)$ & $590(46.9)$ & $848(39.5)$ & \\
\hline$\geq 50$ & $5184(28.0)$ & $3938(26.1)$ & $431(34.2)$ & $815(38.0)$ & \\
\hline Family-care needs & 9889 (47.1) & $8500(49.6)$ & $507(35.3)$ & $882(36.7)$ & $<0.001^{*}$ \\
\hline Any of the following & $1856(9.5)$ & $1358(8.5)$ & $163(12.2)$ & $335(14.8)$ & $<0.001^{*}$ \\
\hline Hyperlipidemia & 898 (4.6) & $674(4.2)$ & $77(5.8)$ & $147(6.5)$ & $<0.001^{*}$ \\
\hline Hyperglycemia & $541(2.8)$ & $429(2.7)$ & $36(2.7)$ & $76(3.3)$ & 0.192 \\
\hline Hypertension & $931(4.8)$ & $644(4.0)$ & $92(6.9)$ & $195(8.6)$ & $<0.001^{*}$ \\
\hline
\end{tabular}

$B M I$ body mass index, $D R$ delivery room, $E R$ emergency room, $I C U$ intensive care unit, $O P D$ outpatient clinic, $O R$ operating room Multiple units indicated that participants worked in more than one of the listed units

Percentages were calculated based on the total number of non-missing cases

and HNs. Scores for stress from the impact of and fatigue from work performance were significantly higher among $\mathrm{CNs}$ and NPs than HNs. Promotion-induced stress was higher among CNs than among HNs and NPs. Finally, NPs showed higher mean scores for other sources of stress than did CNs and HNs. 
Table 2 Sources of work stress for the different nursing positions; $\boldsymbol{n}=\mathbf{2 0 , 9 9 6}$

\begin{tabular}{|c|c|c|c|c|c|c|}
\hline Sources of stress & $\begin{array}{l}\text { Clinical } \\
\text { registered } \\
\text { Nurses } \\
(n=17,152) \\
\text { Mean (SD) }\end{array}$ & $\begin{array}{l}\text { Nurse } \\
\text { practitioners } \\
(n=1438) \\
\text { Mean (SD) }\end{array}$ & $\begin{array}{l}\text { Head } \\
\text { Nurses } \\
(n=2406) \\
\text { Mean } \\
(\text { SD) }\end{array}$ & $p$ value & Post hoc & Aspects \\
\hline Overall level of work stress & $69.52(15.53)$ & $70.36(14.51)$ & $\begin{array}{l}69.46 \\
(15.42)\end{array}$ & 0.188 & - & - \\
\hline Evaluation & $3.33(1.16)$ & $3.36(1.14)$ & $4.01(1.07)$ & $\begin{array}{l}< \\
0.001\end{array}$ & $\begin{array}{l}\mathrm{HN}>\mathrm{CN}= \\
\mathrm{NP}\end{array}$ & 1 \\
\hline Health department or other external policy requirements & $3.17(1.13)$ & $3.09(1.10)$ & $3.71(1.10)$ & $\begin{array}{l}< \\
0.001\end{array}$ & $\begin{array}{l}\mathrm{HN}>\mathrm{CN}> \\
\mathrm{NP}\end{array}$ & 1 \\
\hline Colleagues from own department & $2.70(0.91)$ & $2.71(0.90)$ & $2.79(0.90)$ & $\begin{array}{l}< \\
0.001\end{array}$ & $\begin{array}{l}\mathrm{HN}>\mathrm{CN}= \\
\mathrm{NP}\end{array}$ & 2 \\
\hline Hospital management & $2.83(1.05)$ & $2.88(1.03)$ & $3.04(1.03)$ & $\begin{array}{l}< \\
0.001\end{array}$ & $\begin{array}{l}\mathrm{HN}>\mathrm{CN}= \\
\mathrm{NP}\end{array}$ & 2 \\
\hline Too many extra-occupational duties & $3.36(1.00)$ & $3.31(1.00)$ & $3.46(1.00)$ & $\begin{array}{l}< \\
0.001\end{array}$ & $\begin{array}{l}\mathrm{HN}>\mathrm{CN}= \\
\mathrm{NP}\end{array}$ & 2 \\
\hline Performance requirements & $2.94(1.00)$ & $2.94(1.00)$ & $3.09(1.00)$ & $\begin{array}{l}< \\
0.001\end{array}$ & $\begin{array}{l}\mathrm{HN}>\mathrm{CN}= \\
\mathrm{NP}\end{array}$ & 2 \\
\hline Supervisor of own department & $2.98(0.89)$ & $3.04(0.91)$ & $3.04(0.89)$ & $\begin{array}{l}< \\
0.001\end{array}$ & $\begin{array}{l}\mathrm{NP}=\mathrm{HN}> \\
\mathrm{CN}\end{array}$ & 3 \\
\hline Other departmental supervisors or colleagues & $2.54(0.93)$ & $2.63(0.93)$ & $2.58(0.88)$ & 0.008 & $\begin{array}{l}\mathrm{NP}>\mathrm{CN}= \\
\mathrm{HN}\end{array}$ & 4 \\
\hline Work difficulty and complexity & $3.24(0.88)$ & $3.32(0.85)$ & $3.25(0.88)$ & $\begin{array}{l}< \\
0.001\end{array}$ & $\begin{array}{l}\mathrm{NP}>\mathrm{CN}= \\
\mathrm{HN}\end{array}$ & 4 \\
\hline Uncertainty regarding medical results or work outcomes & $3.26(0.91)$ & $3.36(0.90)$ & $3.26(0.91)$ & $\begin{array}{l}< \\
0.001\end{array}$ & $\begin{array}{l}\mathrm{NP}>\mathrm{CN}= \\
\mathrm{HN}\end{array}$ & 4 \\
\hline Urgency of work & $3.13(0.98)$ & $3.19(0.98)$ & $3.13(1,00)$ & 0.003 & $\begin{array}{l}\mathrm{NP}>\mathrm{CN}= \\
\mathrm{HN}\end{array}$ & 4 \\
\hline Insufficient work leadership or support & $2.80(0.93)$ & $2.92(0.95)$ & $2.72(0.93)$ & 0.001 & $\begin{array}{l}\mathrm{NP}>\mathrm{CN}> \\
\mathrm{HN}\end{array}$ & 5 \\
\hline Lack of job security & $2.88(1.03)$ & $3.28(1.04)$ & $2.65(1.01)$ & $\begin{array}{l}< \\
0.001\end{array}$ & $\begin{array}{l}\mathrm{NP}>\mathrm{CN}> \\
\mathrm{HN}\end{array}$ & 5 \\
\hline Unequal work requirements & $3.04(1.05)$ & $3.15(1.04)$ & $2.94(1.05)$ & $\begin{array}{l}< \\
0.001\end{array}$ & $\begin{array}{l}\mathrm{NP}>\mathrm{CN}> \\
\mathrm{HN}\end{array}$ & 5 \\
\hline Patients & $3.13(0.87)$ & $3.10(0.83)$ & $2.82(0.88)$ & $\begin{array}{l}< \\
0.001\end{array}$ & $\begin{array}{l}\mathrm{CN}=\mathrm{NP}> \\
\mathrm{HN}\end{array}$ & 6 \\
\hline Workload or working hours & $3.36(0.89)$ & $3.36(0.87)$ & $3.33(0.90)$ & $\begin{array}{l}< \\
0.001\end{array}$ & $\begin{array}{l}C N=N P> \\
H N\end{array}$ & 6 \\
\hline Labor burden & $3.33(0.92)$ & $3.31(0.90)$ & $3.25(0.94)$ & $\begin{array}{l}< \\
0.001\end{array}$ & $\begin{array}{l}\mathrm{CN}=\mathrm{NP}> \\
\mathrm{HN}\end{array}$ & 6 \\
\hline Job hazards & $3.23(0.95)$ & $3.20(0.92)$ & $2.97(0.95)$ & $\begin{array}{l}< \\
0.001\end{array}$ & $\begin{array}{l}C N=N P> \\
H N\end{array}$ & 6 \\
\hline Contact with death or similar experiences during work & $3.15(1.01)$ & $3.09(0.95)$ & $2.84(1.00)$ & $\begin{array}{l}< \\
0.001\end{array}$ & $\begin{array}{l}C N=N P> \\
H N\end{array}$ & 6 \\
\hline $\begin{array}{l}\text { Unfamiliarity with work, insufficient training, or not applying one's } \\
\text { knowledge }\end{array}$ & $2.83(0.93)$ & $2.81(0.92)$ & $2.60(0.90)$ & $\begin{array}{l}< \\
0.001\end{array}$ & $\begin{array}{l}C N=N P> \\
H N\end{array}$ & 6 \\
\hline Promotion stress & $2.92(1.02)$ & $2.70(0.98)$ & $2.69(1.01)$ & $\begin{array}{l}< \\
0.001\end{array}$ & $\begin{array}{l}\mathrm{CN}>\mathrm{HN}= \\
\mathrm{NP}\end{array}$ & 7 \\
\hline Health insurance & $2.55(1.07)$ & $2.71(1.08)$ & $2.52(1.10)$ & $\begin{array}{l}< \\
0.001\end{array}$ & $\begin{array}{l}\mathrm{NP}>\mathrm{CN}= \\
\mathrm{HN}\end{array}$ & 8 \\
\hline Lack of mental support & $2.82(0.97)$ & $2.90(0.95)$ & $2.77(0.97)$ & 0.003 & $\begin{array}{l}\mathrm{NP}>\mathrm{CN}= \\
\mathrm{HN}\end{array}$ & 8 \\
\hline
\end{tabular}

Note: The eight sources of work stress are: (1) evaluation and policy; (2) colleagues, higher-level officers, and extra-occupational duties; (3) supervisor of own department; (4) work content and opinions of individuals from other departments; (5) lack of work leadership, security, and equality; (6) impact of and fatigue from work performance; (7) promotion; and (8) others 
Factors associated with hyperlipidemia, hyperglycemia, and hypertension among nurses were examined using logistic regression (Table 3). Crude odds ratio (OR) showed that NPs were 1.867-times more likely to have hyperlipidemia, hyperglycemia, and hypertension than were $\mathrm{CNs}$ (95\% confidence interval [CI]: 1.642-2.124), and HNs were 1.501-times more likely to have hyperlipidemia, hyperglycemia, and hypertension than were CNs (95\% CI: 1.262-1.784). Level of work stress correlated significantly with hyperlipidemia, hyperglycemia, and hypertension; a one-point increase in work stress generated a 1.008-times increase in risk of hyperlipidemia, hyperglycemia, and hypertension (95\% CI: 1.004-1.011).

After controlling for other variables in the model, no significant differences were found among the nursing positions regarding the ORs for hyperlipidemia, hyperglycemia, and hypertension (adjusted model 1 and 2 in Table 3). Regarding demographic variables (adjusted

Table 3 Logistic regression models for factors associated with hyperlipidemia, hyperglycemia, and hypertension

\begin{tabular}{|c|c|c|c|c|}
\hline & $\begin{array}{l}\text { Unadjusted model } \\
\text { OR }(95 \% \mathrm{CI})\end{array}$ & $\begin{array}{l}\text { Adjusted model } 1 \\
\text { OR (95\% Cl) }\end{array}$ & $\begin{array}{l}\text { Adjusted model } 2 \\
\text { OR }(95 \% \mathrm{Cl})\end{array}$ & $\begin{array}{l}\text { Adjusted model } 3 \\
\text { OR }(95 \% \mathrm{CI})\end{array}$ \\
\hline \multicolumn{5}{|l|}{ Positions (clinical registered nurses ${ }^{a}$ ) } \\
\hline Nurse practitioners & $1.867(1.642-2.124)^{* * *}$ & $0.883(0.738-1.057)$ & $0.847(0.695-1.032)$ & $0.849(0.696-1.035)$ \\
\hline Head nurses & $1.501(1.262-1.784)^{* * *}$ & $1.021(0.803-1.297)$ & $0.966(0.743-1.258)$ & $0.967(0.740-1.265)$ \\
\hline \multicolumn{5}{|l|}{ Age $\left(<26\right.$ years $\left.^{\mathrm{a}}\right)$} \\
\hline $26-35$ & $1.711(1.406-2.081)^{* * *}$ & $1.673(1.251-2.239)^{* * *}$ & $1.746(1.271-2.399)^{* * *}$ & $1.759(1.280-2.418)^{* * *}$ \\
\hline $36-45$ & $4.475(3.692-5.423)^{* * *}$ & $4.125(3.007-5.659)^{* * *}$ & $4.468(3.174-6.288)^{* * *}$ & $4.605(3.260-6.506)^{* * *}$ \\
\hline $46-55$ & $11.006(8.942-13.547)^{* * *}$ & $9.856(6.964-13.950)^{* * *}$ & $11.197(7.685-16.316)^{* * *}$ & $11.718(8.006-17.150)^{* * *}$ \\
\hline$>55$ & $19.119(12.981-28.158)^{* * *}$ & $16.361(9.303-28.773)^{* * *}$ & $25.143(13.135-48.132)^{* * *}$ & $26.149(13.632-50.161)^{* * *}$ \\
\hline \multicolumn{5}{|l|}{ Gender (male $)^{a}$} \\
\hline Female & $1.529(1.140-2.051)^{* *}$ & $1.442(0.945-2.199)$ & $1.669(1.080-2.577)^{*}$ & $1.674(1.080-2.594)^{*}$ \\
\hline \multicolumn{5}{|l|}{ BMI $\left(<18.5^{a}\right)$} \\
\hline $18.5-23.9$ & $1.868(1.467-2.378)^{* * *}$ & $1.262(0.950-1.675)$ & $1.301(0.948-1.786)$ & $1.305(0.950-1.791)$ \\
\hline$\geq 24$ & $4.975(3.904-6.341){ }^{* * *}$ & $3.242(2.438-4.311)^{* * *}$ & $3.353(2.439-4.612)^{* * *}$ & $3.364(2.446-4.627)^{* * *}$ \\
\hline \multicolumn{5}{|l|}{ Marital status (never married ${ }^{\mathrm{a}}$ ) } \\
\hline Married & $2.137(1.928-2.370)^{* * *}$ & $1.122(0.947-1.330)$ & $1.117(0.927-1.347)$ & $1.121(0.930-1.352)$ \\
\hline Divorced or widowed & $2.891(2.245-3.722)^{* * *}$ & $1.203(0.857-1.689)$ & $1.143(0.782-1.669)$ & $1.140(0.780-1.666)$ \\
\hline \multicolumn{5}{|l|}{ Job experience $\left.(<5 \text { years })^{\mathrm{a}}\right)$} \\
\hline $5-9$ years & $2.274(1.572-3.287)^{* * *}$ & $1.296(0.814-2.064)$ & $1.183(0.712-1.966)$ & $1.208(0.726-2.009)$ \\
\hline 10-14 years & $1.698(1.435-2.008)^{* * *}$ & $1.052(0.835-1.326)$ & $1.003(0.778-1.292)$ & $1.017(0.789-1.312)$ \\
\hline$\geq 15$ years & $2.495(2.199-2.831)^{* * *}$ & $1.147(0.947-1.390)$ & $1.100(0.893-1.355)$ & $1.114(0.904-1.374)$ \\
\hline \multicolumn{5}{|l|}{ Work unit $\left(O P D^{a}\right)$} \\
\hline ER/ICU & $0.599(0.509-0.704)^{* * *}$ & $0.964(0.769-1.210)$ & $1.004(0.782-1.290)$ & $1.021(0.793-1.313)$ \\
\hline General ward & $0.618(0.535-0.714)^{* * *}$ & $0.972(0.792-1.192)$ & $0.968(0.773-1.212)$ & $0.988(0.787-1.240)$ \\
\hline OR/DR & $0.910(0.763-1.085)$ & $0.981(0.767-1.255)$ & $0.863(0.652-1.142)$ & $0.866(0.654-1.146)$ \\
\hline Multiple units & $0.890(0.744-1.064)$ & $0.844(0.659-1.081)$ & $0.815(0.622-1.069)$ & $0.827(0.629-1.088)$ \\
\hline \multicolumn{5}{|l|}{ Weekly work hours $\left(\leq 40 h^{\mathrm{a}}\right)$} \\
\hline $41-49 h$ & $1.093(0.964-1.238)$ & $1.086(0.928-1.270)$ & $1.050(0.885-1.247)$ & $1.059(0.892-1.258)$ \\
\hline$\geq 50 \mathrm{~h}$ & $1.141(0.995-1.307)$ & $1.237(1.041-1.471)^{*}$ & $1.133(0.937-1.370)$ & $1.157(0.955-1.402)$ \\
\hline Family care needs & $0.905(0.822-0.996)^{*}$ & $1.179(1.017-1.366)^{*}$ & $1.156(0.982-1.360)$ & $1.148(0.975-1.352)$ \\
\hline Stress level & $1.008(1.004-1.011)^{* * *}$ & - & $1.011(1.006-1.016)^{* * *}$ & $1.011(1.006-1.017)^{* * *}$ \\
\hline Interaction term (NPs $\times$ stress level) & - & - & - & $0.997(0.984-1.010)$ \\
\hline Interaction term (HNs $\times$ stress level) & - & - & - & $0.999(0.983-1.016)$ \\
\hline
\end{tabular}

${ }^{a}$ Reference

$p<0.05^{*}, p<0.01^{* *}, p<0.001^{* * *}$

Adjusted model 1 was adjusted for age, gender, marital status, work experience, work unit, weekly work hours, and family-care needs

Adjusted model 2 was adjusted for age, gender, marital status, work experience, work unit, weekly work hours, family-care needs, and stress level

Adjusted model 3 was adjusted for age, gender, marital status, work experience, work unit, weekly work hours, family-care needs, stress level, and interaction term 
model 1), participants aged $\geq 55,46-54,36-45$, and $26-$ 35 years were 16.361 - (95\% CI: 9.303-28.773), 9.856(95\% CI: 6.964-13.950), 4.125- (95\% CI: 3.007-5.659), and 1.673-times (95\% CI: 1.251-2.239), respectively, more likely to have hyperlipidemia, hyperglycemia, and hypertension than those aged under 26 years. Nurses with a BMI of $\geq 24 \mathrm{~kg} / \mathrm{m}^{2}$ were 1.237 -times (95\% CI: 1.041-1.471) more likely to have hyperlipidemia, hyperglycemia, and hypertension than those with a BMI of < $24 \mathrm{~kg} / \mathrm{m}^{2}$. Nurses who worked over $50 \mathrm{~h}$ a week were 1.237-times (95\% CI: 1.041-1.471) more likely to have hyperlipidemia, hyperglycemia, and hypertension than those who worked $40-49 \mathrm{~h}$ a week. Finally, nurses who had family-care needs were 1.179-times (95\% CI: 1.0171.366) more likely to have hyperlipidemia, hyperglycemia, and hypertension than those without family-care needs.

In adjusted model 2, we added level of work stress to adjusted model 1 . Level of work stress was significantly associated with hyperlipidemia, hyperglycemia, and hypertension; every one-point increase in work stress corresponded to a 1.011-times increase in the OR of hyperlipidemia, hyperglycemia, and hypertension (95\% CI: 1.006-1.016). After adding level of work stress, both weekly work hours and family-care needs lost their significance. These results suggested that the effect of extended work hours $(\geq 50 \mathrm{~h})$ and family-care needs on hyperlipidemia, hyperglycemia, and hypertension can be explained by perceived level of work stress. After adding level of work stress to the model, we found that women were 1.669-times (95\% CI: $1.080-2.577$ ) more likely to have hyperlipidemia, hyperglycemia, and hypertension than men. Age and BMI remained risk factors for hyperlipidemia, hyperglycemia, and hypertension. The results of adjusted model 3 showed that there was no significant interaction between job position and level of work stress in regard to hyperlipidemia, hyperglycemia, and hypertension, meaning the association between work stress and hyperlipidemia, hyperglycemia, and hypertension did not differ among HNs, NPs, and CNs.

We further used logistic regression to analyze whether the association between work stress and hyperlipidemia, hyperglycemia, and hypertension differed across sources of work stress (Table 4). The adjusted model (Table 4) showed that all eight sources of stress were significantly associated with hyperlipidemia, hyperglycemia, and hypertension. Their CIs overlapped, indicating that the association between work stress and hyperlipidemia, hyperglycemia, and hypertension did not significantly differ across the sources of work stress.

\section{Discussion}

The present research found significant differences among HNs, NPs, and CNs regarding sources of
Table 4 Logistic regression results for the effect of work stress from different sources on hyperlipidemia, hyperglycemia, and hypertension

\begin{tabular}{|c|c|}
\hline Source of stress & OR $(95 \% \mathrm{Cl})$ \\
\hline Adjusted model (aspects 1) & $1.083(1.048-1.118)^{* * *}$ \\
\hline Adjusted model (aspects 2) & $1.054(1.030-1.079){ }^{* * *}$ \\
\hline Adjusted model (aspects 3) & $1.117(1.040-1.200)^{* * *}$ \\
\hline Adjusted model (aspects 4) & $1.040(1.016-1.064){ }^{* * *}$ \\
\hline Adjusted model (aspects 5) & $1.054(1.028-1.081)^{* * *}$ \\
\hline Adjusted model (aspects 6) & $1.032(1.016-1.049)^{* * *}$ \\
\hline Adjusted model (aspects 7) & $1.104(1.036-1.177){ }^{* * *}$ \\
\hline Adjusted model (aspects 8) & $1.083(1.042-1.126)^{* * *}$ \\
\hline \multicolumn{2}{|c|}{$\begin{array}{l}\text { Note: Eight aspects: (1) evaluation and policy, (2) colleagues, higher-level } \\
\text { officers, and extra-occupational duties, (3) supervisor of own department, (4) } \\
\text { opinions of individuals from other departments, (5) lack of work leadership, } \\
\text { security, and equality, (6) impact of and fatigue from work execution, (7) } \\
\text { promotion, (8) others } \\
\text { Model was adjusted for age, gender, job positions, marital status, work } \\
\text { experience, work unit, weekly work hours, and family care needs }\end{array}$} \\
\hline
\end{tabular}

perceived work stress; these differences in stressors were determined to be associated with the different work contents of the respective job positions. Our results showed that evaluation, hospital management, and communication with others is the primary stressor for HNs, that work complexity, uncertainty regarding medical results, urgency of work, and lack of job security and support is the primary stressor for NPs, and that promotion is the primary stressor for CNs. Hospitals in Taiwan must undergo evaluations every three years to certify/retain their status as medical centers, regional hospitals, district hospitals, and/or teaching hospitals. In addition, there are several other types of external evaluations/certifications that hospitals can seek to underline their high standards and quality of care [30]. HNs are the head administrators in departments of nursing, and are usually the leaders of unit evaluations; this explains why evaluation and management-related aspects represented the primary stressor for this group [31]. On the other hand, NPs and CPs experience most stress from heavy workloads or extended working hours, which may be due to their engagement in shift work and workforce shortages. Workforce shortages could increase nurses' working hours, reduce some of their off hours, induced burnout, and exacerbate workplace bullying [32]. Further, workforce shortages and heavy workloads in the nursing sector have become core issues in Taiwan's national policies. Although existing policies in Taiwan stipulate that nurse-patient ratios should not exceed 1:9 in medical centers, 1:12 in regional hospitals, and 1:15 in district hospitals [33], these ratios are still higher than the corresponding ratios in many developed countries, such as the US, Japan, and European countries [34, 35]. An excessive nurse-patient ratio may cause occupational 
burnout and turnover intentions as well as an increased risk of medication errors and a growth in patient mortality rates, ulcers, infection, and pneumonia [32, 34, 36]. Thus, when drafting stress-management solutions for nurses, hospitals should consider the diverse influences of different sources of stress and job positions.

Although the significance of each source of stress varied across the different nursing positions, the overall work-stress perception did not significantly differ across the sample. The prevalence of hyperlipidemia, hyperglycemia, and hypertension differed across job positions, being highest among HNs, followed by NPs and CNs. Through univariate analysis, we observed distributional differences among the three nursing positions in terms of age, gender, BMI, marital status, years of work experience, work unit, work hours per week, and family-care needs. In sum, nurses in all three job positions had distinct characteristics, and these attributes, rather than job position, influenced prevalence of hyperlipidemia, hyperglycemia, and hypertension.

Our finding regarding factors related to hyperlipidemia, hyperglycemia, and hypertension agree with previous findings. Older individuals have a higher risk of suffering from hyperlipidemia, hyperglycemia, and hypertension [9, 37]. Excessive BMI also has a significant correlation with hyperlipidemia, hyperglycemia, and hypertension [36, 38]; the present study defined obesity as BMI $\geq 24$ in accordance with the criteria in Taiwan; among the participants, $33.0 \%$ of $\mathrm{HNs}$ were obese, followed by NPs (26.7\%) and CNs (25.2\%). The HNs' average age was higher than those of the other groups, and they mainly performed administrative work. Further, most NPs and CNs worked on the front line, which entails more physically strenuous tasks. Regarding the correlation of hyperlipidemia, hyperglycemia, and hypertension with working hours, the longer the working hours, the higher the risk of developing hyperlipidemia, hyperglycemia, and hypertension [39]. Finally, regarding the finding that participants who had familycare needs also showed a higher risk of hyperlipidemia, hyperglycemia, and hypertension, previous research has reported that individuals with family-care needs have a stronger stress perception than do those without such needs [40], which would further impact hyperlipidemia, hyperglycemia, and hypertension [41].

Our study revealed that an increased level of perceived stress is associated with an increased OR for hyperlipidemia, hyperglycemia, and hypertension, which is consistent with the literature [3-5]. Stress perception is likely to lead to the adoption of harmful lifestyles, such as smoking, alcoholism, high-sugar and high-fat diets, and insufficient exercise [12, 42]. Without early prevention, job stress and an unhealthy lifestyle can not only increase the risk of hyperlipidemia, hyperglycemia, and hypertension, but also lead to cardiovascular diseases $[8,4]$. Our results suggest that nursing management in hospitals should utilize appropriate stress management strategies and plan stress reducing interventions. There is a need for effective stress relieving and health enhancing lifestyle programs for nurses in hospitals.

\section{Limitations}

This study used self-report data, and we were unable to verify the accuracy of the participants' reports. No objective measurement of hyperlipidemia, hyperglycemia, and hypertension was available, and under-reporting was possible; however, the rate of under-reporting across the three groups could be non-differential. Finally, causal relationships could not be inferred as a result of the use of a cross-sectional design.

\section{Conclusions}

Medium to high job stress was observed in nurses. Although stressors varied by nursing position, the overall stress level was similar across HNs, NPs, and CNs. Higher job stress levels, increased age, higher BMI, long work hours, and caring for family members were positively associated with hyperlipidemia, hyperglycemia, and hypertension. Further studies are needed to identify and verify effective strategies for reducing work stress in nursing staff.

\section{Abbreviations \\ US: United States; NP: Certified nurse practitioner; CN: Clinical registered nurse; HN: Head nurse/ nurse manager; ANOVA: Analysis of variance; \\ SD: Standard deviation; BMl: Body mass index; OR: Odds ratio; $\mathrm{Cl}$ : Confidence interval; DR: Delivery room; ER: Emergency room; ICU: Intensive care unit; OPD: Outpatient clinic; OR: Operating room}

\section{Acknowledgments}

The survey was commissioned by the Health Promotion Administration, Department of Health, Taiwan. We thank the Health Promotion Administration for granting us to use the data.

\section{Authors' contributions}

PYC, STC and LYC conceived and designed the study. PYC analyzed the data. PYC and LYC drafted the manuscript. PYC, WYL, NH, STC, and LYC interpreted the data and substantively revised the manuscript. All authors reviewed and approved the final manuscript.

\section{Funding}

The publication fee for this article was supported by the Ministry of Science and Technology grant number MOST108-2314-B-010-059-MY3.

\section{Availability of data and materials}

The data that support the findings of this study are available from the Health Promotion Administration, Taiwan. Restrictions apply to the availability of these data, which were used under license for this study. Data are available with the permission of the Health Promotion Administration, Taiwan.

\section{Declarations}

Ethics approval and consent to participate

The study protocol was approved by the Institutional Review Board of the Health Promotion Administration, Taiwan (HPA investigation number: 
EC1030308-F-W). Written informed consent was obtained from all participants. The research has been performed in accordance with the Declaration of Helsinki.

\section{Consent for publication}

Not applicable.

\section{Competing interests}

The authors have no conflicts of interest to declare.

\section{Author details}

'Department of Surgery, National Taiwan University Hospital, Taipei, Taiwan. ${ }^{2}$ Institute of Public Health, National Yang Ming Chaio Tung University, Yang-Ming Campus, Taipei, Taiwan. ${ }^{3}$ Center for Healthcare Quality Management, Cheng Hsin General Hospital, Taipei, Taiwan. ${ }^{4}$ Department of Nursing, National Yang Ming Chiao Tung University, Yang-Ming Campus, Taipei, Taiwan. ${ }^{5}$ Institute of Hospital and Health Care Administration, National Yang Ming Chiao Tung University, Yang-Ming Campus, Taipei, Taiwan. ${ }^{6}$ Institute of Community Health Care, College of Nursing, National Yang Ming Chiao Tung University, Yang-Ming Campus, 155 Li-Nong Street, Section 2, Bei-Tou, Taipei 11221, Taiwan.

\section{Received: 12 October 2021 Accepted: 30 November 2021}

\section{Published online: 13 December 2021}

\section{References}

1. Martín-Del-Río B, Solanes-Puchol Á, Martínez-Zaragoza F, Benavides-Gil G. Stress in nurses: The 100 top-cited papers published in nursing journals. J Adv Nurs. 2018;74(7):1488-504. https://doi.org/10.1111/jan.13566.

2. A MV. Scoping the common antecedents of job stress and job satisfaction for nurses (2000-2013) using the job demands-resources model of stress. J Nurs Manag. 2016;24(2):112-36. https://doi.org/10.1111/jonm.12326.

3. Djindjic $N$, Jovanovic J, Djindjic $B$, Jovanovic $M$, Jovanovic JJ. Associations between the occupational stress index and hypertension, type 2 diabetes mellitus and lipid disorders in middle-aged men and women. Ann Occup Hyg. 2012;56(9):1051-62. https://doi.org/10.1093/annhyg/mes059.

4. Kivimäki M, Kawachi I. Work stress as a risk factor for cardiovascular disease. Curr Cardiol Rep. 2015;17(9):630. https://doi.org/10.1007/s11886-015-0630-8

5. Liu MY, Li N, Li WA, Khan H. Association between psychosocial stress and hypertension: a systematic review and meta-analysis. Neurol Res. 2017;39(6): 573-80. https://doi.org/10.1080/01616412.2017.1317904.

6. Leal JAL, Melo CMM. Nurses' work process in different countries: An integrative review. Rev Bras Enferm. 2018;71(2):413-23. https://doi.org/10.1 590/0034-7167-2016-0468.

7. Navinés R, Martín-Santos R, Olivé V, Valdés M. Work-related stress: Implications for physical and mental health. Med Clin. 2016;146(8):359-66. https://doi.org/10.1016/j.medcli.2015.11.023.

8. Dragano N, Siegrist J, Nyberg ST, Lunau T, Fransson El, Alfredsson L, et al. Effort-reward imbalance at work and incident coronary heart disease: A Multicohort study of 90,164 individuals. Epidemiology. 2017;28(4):619-26. https://doi.org/10.1097/EDE.0000000000000666.

9. Assadi SN. What are the effects of psychological stress and physical work on blood lipid profiles? Medicine. 2017;96(18):1-6. https://doi.org/10.1097/MD. 0000000000006816.

10. Lin PY, Wang JY, Tseng P, Shih DP, Yang CL, Liang WM, et al. Environmental tobacco smoke (ETS) and hyperlipidemia modified by perceived work stress. PLoS One. 2020;15(1):1-12. https://doi.org/10.1371/journal.pone.0227348.

11. Carneiro-Nascimento S, Opacka-Juffry J, Costabile A, Boyle CN, Herde AM, Ametamey SM, et al. Chronic social stress in mice alters energy status including higher glucose need but lower brain utilization. Psychoneuroendocrinology. 2020: 119. https://doi.org/10.1016/j.psyneuen.2020.104747.

12. Gheisari Z, Beiranvand R, Karimi A, Ghalavandi S, Soleymani A, Madmoli M, et al. Relationship between occupational stress and cardiovascular risk factors determination: A case-control study. J Res Med Dent Sci. 2018;6(3): 287-93. https://doi.org/10.24896/jrmds.20186344.

13. Cosgrove MP, Sargeant LA, Caleyachetty R, Griffin SJ. Work-related stress and Type 2 diabetes: Systematic review and meta-analysis. Occup Med. 2012;62(3):167-73. https://doi.org/10.1093/occmed/kqs002.

14. Landsbergis PA, Dobson M, Koutsouras G, Schnall P. Job strain and ambulatory blood pressure: A meta-analysis and systematic review. Am J Public Health. 2013;103(3):61-71. https://doi.org/10.2105/AJPH.2012.301153.
15. Trudel X, Brisson C, Gilbert-Ouimet M, Milot A. Psychosocial stressors at work and ambulatory blood pressure. Curr Cardiol Rep. 2018;20(12):1-9. https:// doi.org/10.1007/s11886-018-1070-z.

16. Luan X, Wang P, Hou W, Chen L, Lou F. Job stress and burnout: A comparative study of senior and head nurses in China. Nurs Health Sci. 2017:19(2):163-9. https://doi.org/10.1111/nhs.12328.

17. Jordan TR, Khubchandani J, Wiblishauser M. The impact of perceived stress and coping adequacy on the health of nurses: A pilot investigation. Nurs Res Pract. 2016;2016:e5342856. https://doi.org/10.1155/2016/5843256.

18. Su SY, Chiou ST, Huang N, Huang CM, Chiang JH, Chien LY. Association between Pap smear screening and job stress Taiwanese nurses. Eur J Oncol Nurs. 2016;20:119-24. https://doi.org/10.1016/j.ejon.2015.07.001.

19. Kakemam E, Raeissi $P$, Raoofi S, Soltani A, Sokhanvar M, Visentin D, et al. Occupational stress and associated risk factors among nurses: A crosssectional study. Contemp Nurse. 2019:55(2-3):237-49. https://doi.org/10.1 080/10376178.2019.1647791.

20. American Nurses Association (ANA). Advanced practice registered nurses (APRN). 2016. Available from: https://www.nursingworld.org/practicepolicy/aprn/. Accessed 10 Dec 2020.

21. Taiwan Ministry of Health and Welfare. Statistics of medical care institution's status \& hospital utilization 2019. Dep of Statistics 2020. Available from: https://www.mohw.gov.tw/cp-4932-54834-2.html. Accessed 17 July 2020.

22. Taiwan Ministry of Health and Welfare. Statistics of medical care institution's status \& hospital utilization 2019: Department of Statistics; 2020. Available from: https://www.mohw.gov.tw/cp-137-52878-2.html. Accessed 17 July 2020.

23. Andregård $A C$, Jangland $E$. The tortuous journey of introducing the nurse practitioner as a new member of the healthcare team: A meta-synthesis. Scand J Caring Sci. 2015;29(1):3-14. https://doi.org/10.1111/scs.12120.

24. Brom HM, Melnyk BM, Szalacha LA, Graham M. Nurse Practitioners' role perception, stress, satisfaction, and intent to stay at a Midwestern academic medical center. J Am Assoc Nurse Pract. 2016;28(5):269-76. https://doi.org/1 $0.1002 / 2327-6924.12278$

25. Chen $\mathrm{CH}$, Wang J, Yang CS, Fan JY. Nurse practitioner job content and stress effects on anxiety and depressive symptoms, and self-perceived health status. J Nurs Manag. 2016;24(5):695-704. https://doi.org/10.1111/jonm.12375.

26. Loveridge S. Straight talk: Nurse manager role stress. Nurs Manage. 2017; 48(4):20-7. https://doi.org/10.1097/01.NUMA.0000514058.63745.ad.

27. Van Bogaert P, Adriaenssens J, Dilles T, Martens D, Van Rompaey B, Timmermans O. Impact of role, job, and organizational characteristics on nursing unit managers' work-related stress and well-being. J Clin Nurs. 2014; 70(11):2622-33. https://doi.org/10.1111/jocn.14165.

28. Chiou ST, Chiang JH, Huang N, Wu CH, Chien LY. Health issues among nurses in Taiwanese hospitals: National survey. Int J Nurs Stud. 2013;50(10): 1377-84. https://doi.org/10.1016/j.ijnurstu.2013.01.012.

29. Lo WY, Chien LY, Hwang FM, Huang N, Chiou ST. From job stress to intention to leave among hospital nurses: A structural equation modeling approach. J Adv Nurs. 2018:74(3):677-88. https://doi.org/10.1111/jan.13481.

30. Joint Commission of Taiwan. Accreditation. 2018. Available from: https:// www.jct.org.tw/cp-1127-1106-22fc4-2.html. Accessed 15 Dec 2020.

31. Lin Y, Chuang T, Liu H, Chen Y, Chen F. Exploring job stressors for head nurses and clinical nurses from the perspective of their job characteristics. Taiwan Gong Gong Wei Sheng Za Zhi. 2011;30(3):245-56. Available from: https://search.proquest.com/docview/1018557121?pq-origsite=gschola r\&fromopenview=true. Accessed 20 Nov 2020.

32. Hsiao STS, Ma SC, Guo SL, Kao CC, Tsai JC, Chung MH, et al. The role of workplace bullying in the relationship between occupational burnout and turnover intentions of clinical nurses. Appl Nurs Res. 2021;

33. Laws and Regulations Database of the Republic of China. Medical care act 2019. Available from: https://law.moj.gov.tw/LawClass/LawAll.aspx?pcode= L0020025. Accessed 02 Dec 2020.

34. Aiken LH, Sermeus W, Van den Heede K, Sloane DM, Busse R, McKee M, et al. Patient safety, satisfaction, and quality of hospital care: cross-sectional surveys of nurses and patients in 12 countries in Europe and the United States. BMC Nurs. 2012;344:e1717. https://doi.org/10.1136/bmi.e1717.

35. Nakata Y, Miyazaki S. Nurses' pay in Japan: Market forces vs. institutional constraints. J Clin Nurs. 2011;20(1-2):4-11. https://doi.org/10.1111/j.1365-2 702.2010.03384.x.

36. Driscoll A, Grant MJ, Carroll D, Dalton S, Deaton C, Jones I, et al. The effect of nurse-to-patient ratios on nurse-sensitive patient outcomes in acute specialist units: A systematic review and meta-analysis. Eur J Cardiovasc Nurs. 2018;17(1):6-22. https://doi.org/10.1177/1474515117721561. 
37. Feng RN, Zhao C, Wang C, Niu YC, Li K, Guo FC, et al. BMl is strongly associated with hypertension and waist circumference is strongly associated with type 2 diabetes and dyslipidemia, in northern Chinese adults. J Epidemiol. 2012;22(4):317-23. https://doi.org/10.2188/jea.je20110120.

38. Poorolajal J, Farbakhsh F, Mahjub H, Bidarafsh A, Babaee E. How much excess body weight, blood sugar, or age can double the risk of hypertension? Public Health. 2016;133:14-8. https://doi.org/10.1016/j.puhe.2 015.10.014.

39. Itani O, Kaneita Y, Ikeda M, Kondo S, Murata A, Ohida T. Associations of work hours and actual availability of weekly rest days with cardiovascular risk factors. J Occup Health. 2013;55(1):11-20. https://doi.org/10.1539/joh.120034-oa.

40. Scott LD, Hwang WT, Rogers AE. Impact of multiple care giving roles on fatigue, stress, and work performance among hospital staff nurses. J Nurs Adm. 2006;36(2):86-95. https://doi.org/10.1097/00005110-200602000-00007.

41. Torimoto Sasai Y, Igarashi A, Wada T, Ogata Y, Yamamoto Mitani N. Female family caregivers face a higher risk of hypertension and lowered estimated glomerular filtration rates: A cross-sectional, comparative study. BMC Public Health. 2015;15:177. https://doi.org/10.1186/s12889-015-1519-6.

42. Pelletier JE, Lytle LA, Laska MN. Stress, health risk behaviors and weight status among community college students. Health Educ Behav. 2016;43(2): 139-44. https://doi.org/10.1177/1090198115598983.

\section{Publisher's Note}

Springer Nature remains neutral with regard to jurisdictional claims in published maps and institutional affiliations.

Ready to submit your research? Choose BMC and benefit from:

- fast, convenient online submission

- thorough peer review by experienced researchers in your field

- rapid publication on acceptance

- support for research data, including large and complex data types

- gold Open Access which fosters wider collaboration and increased citations

- maximum visibility for your research: over $100 \mathrm{M}$ website views per year

At BMC, research is always in progress.

Learn more biomedcentral.com/submissions 